\title{
Module structure of rings of integers in octahedral extensions
}

\author{
by \\ M. Godin and B. Sodaïgui (Valenciennes)
}

1. Introduction. For every number field $K, O_{K}$ denotes its ring of integers and $\mathcal{C l}(K)$ its classgroup.

Let $K / k$ be an extension of number fields of degree $n$. The ring $O_{K}$ is a torsion free $O_{k}$-module of rank $n$, so there exists an ideal $I$ of $O_{k}$ such that $O_{K} \simeq O_{k}^{n-1} \oplus I$ as $O_{k}$-modules. The class of $I$ in $\mathcal{C l}(k)$ is called the Steinitz class of $K / k$ or of $O_{K}$, and is denoted by $\mathcal{C} l_{k}\left(O_{K}\right)$ (see [FT, Theorem 13, p. 95]). The structure of $O_{K}$ as an $O_{k}$-module is determined up to isomorphism by its rank and its Steinitz class.

Now, let $\Gamma$ be a finite group and $\Delta$ a normal subgroup of $\Gamma$. We have the following exact sequence:

$$
\Sigma: \quad 1 \rightarrow \Delta \rightarrow \Gamma \rightarrow \Gamma / \Delta \rightarrow 1 .
$$

We fix a Galois extension $E / k$ with Galois group isomorphic to $\Gamma / \Delta$. We denote by $R(E / k, \Sigma)$ (resp. $R_{t}(E / k, \Sigma)$ ) the set of (realizable) classes $c \in \mathcal{C l}(k)$ such that there exists a Galois extension (resp. Galois extension which is at most tamely ramified, i.e. tame) $N / k$, containing $E$, with an isomorphism $\pi$ from $\operatorname{Gal}(N / k)$ to $\Gamma$ and with $E$ being the subfield of $N$ fixed by $\pi^{-1}(\Delta)$, and the Steinitz class of $O_{N}$ equal to $c$.

For $\Delta=\Gamma, R(E / k, \Sigma)$ (resp. $\left.R_{t}(E / k, \Sigma)\right)$ is simply the set of the Steinitz classes of Galois extensions (resp. tame Galois extensions) of $k$ whose Galois group is isomorphic to $\Gamma$; we write $R(k, \Gamma)$ and $R_{t}(k, \Gamma)$ instead of $R(E / k, \Sigma)$ and $R_{t}(E / k, \Sigma)$.

For previous work concerning the determination of $R(E / k, \Sigma)$ and $R_{t}(E / k, \Sigma)$ see [C1, C2, GS]. In [GS], we consider the case of $\Gamma=A_{4}$, the alternating group, and $\Delta$ its subgroup of order 3 ; under the hypothesis that the class number of $k$ is odd, we determine $R(E / k, \Sigma)$ and $R_{t}(E / k, \Sigma)$ and prove that they are subgroups of $\mathcal{C l}(k)$ when $O_{E}$ is a free $O_{k}$-module or the class number of $k$ is not divisible by 3 .

2000 Mathematics Subject Classification: 11R04, 11R29, 11R33. 
When $\Gamma$ is abelian, a consequence of McCulloh's work (see [Mc]) is that $R_{t}(k, \Gamma)$ is a subgroup of $\mathcal{C l}(k)$. In [C3], it is shown that $R_{t}(k, \Gamma)$ is a subgroup of $\mathcal{C l}(k)$ in the situation when $\Gamma$ is a nonabelian group of order $p^{3}$, and $k$ contains the $m$ th roots of unity, where $p$ is an odd prime number and $m$ is the exponent of $\Gamma$. When $\Gamma$ is the quaternion or dihedral group of order 8 , or the alternating (tetrahedral) group $A_{4}$, it is respectively proven in [So1], [So2] and [GS] that $R_{t}(k, \Gamma)=\mathcal{C} l(k)$ (therefore equal to $R(k, \Gamma)$ ) if the class number of $k$ is odd.

In this paper, we are interested in the case where $\Gamma$ is the symmetric (octahedral) group $S_{4}$ on 4 letters which can be defined by the presentation:

$$
\begin{aligned}
S_{4}=\left\langle\mu, \nu, \sigma, \tau: \mu^{2}=\nu^{2}=\sigma^{3}=\tau^{2}=1, \mu \nu=\nu \mu, \tau \sigma \tau\right. & =\sigma^{-1}, \\
\sigma \mu \sigma^{-1} & =\nu, \tau \mu \tau=\nu\rangle,
\end{aligned}
$$

and

$$
\Delta=\langle\mu, \nu\rangle .
$$

The group $S_{4}$ is a semidirect product of $\Delta$ and $\langle\sigma, \tau\rangle$, where $\Delta \simeq \mathbb{Z} / 2 \mathbb{Z} \times$ $\mathbb{Z} / 2 \mathbb{Z}$ and $\langle\sigma, \tau\rangle \simeq D_{3}$ (or $S_{3}$ ), $D_{3}$ being the dihedral group of order 6 . A Galois extension of $k$ is called octahedral if its Galois group is isomorphic to $S_{4}$.

We have $\operatorname{Gal}(E / k) \simeq\langle\sigma, \tau\rangle$, therefore $E / k$ is a dihedral extension of degree 6. In Section 2, we shall prove the following main result:

TheOREM 1.1. Let $k$ be a number field. Let $E / k$ be a dihedral extension of degree 6 . Assume that the class number of $k$ is odd. Then

(i) $R(E / k, \Sigma)=\mathcal{C} l_{k}\left(O_{E}\right)(\mathcal{C l}(k))^{3}$, where $(\mathcal{C l}(k))^{3}$ is the subgroup of third powers of elements of $\mathcal{C l}(k)$. In addition, if $E / k$ is tame then $R_{t}(E / k, \Sigma)=$ $R(E / k, \Sigma)$.

(ii) $R\left(k, S_{4}\right)=R_{t}\left(k, S_{4}\right)=\mathcal{C l}(k)$.

REMARK. The hypothesis that the class number of $k$ is odd comes from an embedding problem.

If the class number of $k$ is not divisible by 3 then $(\mathcal{C l}(k))^{3}=\mathcal{C l}(k)$. According to the definition of the Steinitz class, $O_{E}$ is a free $O_{k}$-module if and only if $\mathcal{C} l_{k}\left(O_{E}\right)=1$. Therefore we have:

COROLlary 1.2. Under the hypotheses and notation of Theorem 1.1 we have the following assertions:

(1) If the class number of $k$ is not divisible by 3 then $R(E / k, \Sigma)=\mathcal{C l}(k)$ $\left(=R_{t}(E / k, \Sigma)\right.$ if $E / k$ is tame $)$.

(2) If $O_{E}$ is a free $O_{k}$-module then $R(E / k, \Sigma)$ is the subgroup of $\mathcal{C l}(k)$ equal to $(\mathcal{C l}(k))^{3}\left(=R_{t}(E / k, \Sigma)\right.$ if $E / k$ is tame $)$. 
Now we point out our principal motivation for studying the set of Steinitz classes. Let $\mathcal{M}$ be a maximal $O_{k}$-order in $k[\Gamma]$ containing $O_{k}[\Gamma]$ and let $\mathcal{C} \ell(\mathcal{M})$ be its locally free classgroup. We denote by $\mathcal{R}(\mathcal{M})$ the set of realizable classes, that is, the set of classes $c \in \mathcal{C} \ell(\mathcal{M})$ such that there exists a Galois extension $N / k$, at most tamely ramified, and with Galois group isomorphic to $\Gamma$, for which the class of $\mathcal{M} \otimes_{O_{k}[\Gamma]} O_{N}$ is equal to $c$. An interesting problem is to determine the structure of $\mathcal{R}(\mathcal{M})$ in the case that $\Gamma$ is nonabelian, the abelian case being solved by McCulloh (see [Mc]). For instance, in [So2, So3], a close link is shown between the determination of that structure and the problem of studying the Steinitz classes.

2. Proof of the main result. Let $N / k$ be an octahedral extension. If $\pi$ is an isomorphism from $\operatorname{Gal}(N / k)$ to $S_{4}$ and $\gamma \in S_{4}$, one identifies $\pi^{-1}(\gamma)$ with $\gamma$. Let $E / k$ be the subextension of $N$ fixed by $\Delta$. Then $E / k$ is a dihedral extension of degree 6 . Let $k^{\prime} / k$ be the quadratic subextension of $E / k$. Then $N / k^{\prime}$ is a Galois extension with Galois group isomorphic to the alternating group $A_{4}$. The extension $N / E$ is biquadratic, and contains three quadratic extensions of $E$; if $L / E$ is one of these then the others are $\sigma(L)$ and $\sigma^{2}(L)$.

Proposition 2.1. With the above notation we have

$$
\mathcal{C} l_{k}\left(O_{N}\right)=\left(\mathcal{C} l_{k}\left(O_{E}\right)\right)^{4}\left(N_{E / k}\left(\mathcal{C l}_{E}\left(O_{L}\right)\right)\right)^{3} .
$$

Proof (analogous to that in [GS, Proposition 2.1] because $\operatorname{Gal}\left(N / k^{\prime}\right) \simeq$ $A_{4}$ ). By transitivity of the Steinitz class in a tower of number fields (see [F, Theorem 4.1]) we have

$$
\mathcal{C} l_{k}\left(O_{N}\right)=\left(\mathcal{C} l_{k}\left(O_{E}\right)\right)^{4} N_{E / k}\left(\mathcal{C} l_{E}\left(O_{N}\right)\right) .
$$

We know ([GS, Lemme 2.2]) that the Steinitz class of a biquadratic extension is the product of the Steinitz classes of its three quadratic subextensions. Thus

$$
\mathcal{C} l_{E}\left(O_{N}\right)=\mathcal{C} l_{E}\left(O_{L}\right) \mathcal{C} l_{E}\left(O_{\sigma(L)}\right) \mathcal{C} l_{E}\left(O_{\sigma^{2}(L)}\right) .
$$

As we have seen in the proof of [GS, Proposition 2.1], if we write $L=$ $E(\sqrt{m})$, then since $\sigma^{i}(L)=E\left(\sqrt{\sigma^{i}(m)}\right)$ and $\sigma^{i}(\Delta(L / E))=\Delta\left(\sigma^{i}(L) / E\right)$ (where $\Delta(L / E)$ and $\Delta\left(\sigma^{i}(L) / E\right)$ denote the discriminants), we have by Artin (see $[\mathrm{A}]$ )

$$
\mathcal{C} l_{E}\left(O_{\sigma^{i}(L)}\right)=\sigma^{i}\left(\mathcal{C} l_{E}\left(O_{L}\right)\right)
$$

Hence

$$
N_{E / k}\left(\mathcal{C} l_{E}\left(O_{N}\right)\right)=\left(N_{E / k}\left(\mathcal{C} l_{E}\left(O_{L}\right)\right)\right)^{3} .
$$

This completes the proof.

To prove Theorem 1.1, we need the following lemma which is a criterion for an embedding problem. This lemma is well known. Its origin lies in a 
statement in [Ma, p. 365, application for $n=4$, (ii)] without proof. A part of it is Theorem I.2 of [J]. Here we complete the proof.

Lemma 2.2. Let $k$ be a number field. Let $E / k$ be a dihedral extension of degree 6 with Galois group $\langle\sigma, \tau\rangle$, and let $K / k$ be its (cubic non-Galois) subextension fixed by $\tau$. Let $a \in K$ be an element which is not a square in $E$, and let $M$ be the quadratic extension $K(\sqrt{a}) / K$. Then the following assertions are equivalent:

(1) $E / k$ is embeddable in an octahedral extension $N / k$ containing $M$ and such that $N / M$ is biquadratic.

(2) $N_{K / k}(a)$ is a square in $k$, where $N_{K / k}$ is the norm map in $K / k$.

In addition if the embedding is possible, we can choose $N=E(\sqrt{a}, \sqrt{\sigma(a)})$.

Proof. The implication $(1) \Rightarrow(2)$ is Theorem I.2 of $[\mathrm{J}]$. Now we prove $(2) \Rightarrow(1)$. Since $a$ is not a square in $E$, neither is $\sigma(a)$. By Kummer theory and the fact that $N_{K / k}(a)$ is a square, we have $E(\sqrt{a}) / E \neq E(\sqrt{\sigma(a)}) / E$. Let $N / E$ be the biquadratic extension $E(\sqrt{a}, \sqrt{\sigma(a)}) / E$, and $\sigma_{1}$ and $\sigma_{2}$ the generators of $\operatorname{Gal}(N / E)$. We denote by $\bar{\sigma}$ (resp. $\bar{\tau})$ a $k$-embedding of $N$ which extends $\sigma$ (resp. $\tau$ ). It is immediate that $\bar{\sigma}(\sqrt{a})= \pm \sqrt{\sigma(a)}$. As $N_{K / k}(a)=a \sigma(a) \sigma^{2}(a)$ is a square in $k$, we deduce that $\sigma^{2}(a)$ has a square root in $N$. Hence $\bar{\sigma}(\sqrt{\sigma(a)})= \pm \sqrt{\sigma^{2}(a)}$, and $\bar{\sigma}(N) \subset N$. We have $(\sqrt{a})^{2}=a$, so $(\bar{\tau}(\sqrt{a}))^{2}=\tau(a)=a$, and then $\bar{\tau}(\sqrt{a})= \pm \sqrt{a}$. Similarly, $(\bar{\tau}(\sqrt{\sigma(a)}))^{2}=\tau \sigma(a)=\sigma^{2} \tau(a)=\sigma^{2}(a)$, and therefore $\bar{\tau}(\sqrt{\sigma(a)})=$ $\pm \sqrt{\sigma^{2}(a)}$ and $\bar{\tau}(N) \subset N$. We conclude that $N / k$ is Galois of degree 24 and $\operatorname{Gal}(N / k)=\left\langle\sigma_{1}, \sigma_{2}, \bar{\sigma}, \bar{\tau}\right\rangle$. Now, choose (for instance) $\sigma_{1}, \sigma_{2}, \bar{\sigma}, \bar{\tau}$ defined by:

$$
\begin{aligned}
\sigma_{1}(\sqrt{a}) & =-\sqrt{a}, & \sigma_{1}(\sqrt{\sigma(a)}) & =\sqrt{\sigma(a)}, & & \sigma_{1}\left(\sqrt{\sigma^{2}(a)}\right)=-\sqrt{\sigma^{2}(a)}, \\
\sigma_{2}(\sqrt{a}) & =-\sqrt{a}, & \sigma_{2}(\sqrt{\sigma(a)}) & =-\sqrt{\sigma(a)}, & & \sigma_{2}\left(\sqrt{\sigma^{2}(a)}\right)=\sqrt{\sigma^{2}(a)}, \\
\bar{\sigma}(\sqrt{a}) & =\sqrt{\sigma(a)}, & \bar{\sigma}(\sqrt{\sigma(a)}) & =\sqrt{\sigma^{2}(a)}, & & \bar{\sigma}\left(\sqrt{\sigma^{2}(a)}\right)=\sqrt{a} \\
\bar{\tau}(\sqrt{a}) & =\sqrt{a}, & \bar{\tau}(\sqrt{\sigma(a)}) & =\sqrt{\sigma^{2}(a)}, & & \bar{\tau}\left(\sqrt{\sigma^{2}(a)}\right)=\sqrt{\sigma(a)} .
\end{aligned}
$$

An easy calculation shows that $\operatorname{Gal}(N / k) \simeq S_{4}$, which completes the proof.

Proof of Theorem 1.1(i). Let $k$ be a number field. Let $E / k$ be a dihedral extension of degree 6 . Assume that the class number of $k$ is odd. We begin by proving the equalities

$$
\begin{aligned}
R(E / k, \Sigma) & =\left(\mathcal{C} l_{k}\left(O_{E}\right)\right)^{4}\left(N_{E / k}(\mathcal{C l}(E))\right)^{3}, \\
R_{t}(E / k, \Sigma) & =R(E / k, \Sigma) \quad \text { if } E / k \text { is tame. }
\end{aligned}
$$

The inclusion (for any number field $k$ )

$$
R(E / k, \Sigma) \subset\left(\mathcal{C l}_{k}\left(O_{E}\right)\right)^{4}\left(N_{E / k}(\mathcal{C l}(E))\right)^{3}
$$


is an immediate consequence of Proposition 2.1. Let us now show

$$
\left(\mathcal{C} l_{k}\left(O_{E}\right)\right)^{4}\left(N_{E / k}(\mathcal{C l}(E))\right)^{3} \subset R(E / k, \Sigma) .
$$

Let $c \in N_{E / k}(\mathcal{C l}(E))$. Since $N_{E / k}(\mathcal{C l}(E))$ is a subgroup of $\mathcal{C l}(k)$, its order is also odd. Hence there exists $c^{\prime} \in N_{E / k}(\mathcal{C l}(E))$ such that $c=c^{\prime 4}$. Let $C \in \mathcal{C l}(E)$ be such that $c^{\prime}=N_{E / k}(C)$.

We denote by $\mathcal{C l}\left(E, 4 O_{E}\right)$ the ray classgroup modulo $4 O_{E}$. The canonical surjection from $\mathcal{C l}\left(E, 4 O_{E}\right)$ onto $\mathcal{C l}(E)$ and the Chebotarev density theorem in ray classgroups (see [N, Chap. V, Theorem 6.4, p. 132]) allow us to assert that there exist $m \in E^{\times}$, a fractional ideal $I$ of $O_{E}$, and a prime ideal $\mathfrak{P}$ of $O_{E}$ such that $\mathfrak{P} \cap O_{k}$ splits completely in $E / k$ and

$$
m O_{E}=I^{2} \mathfrak{P}, \quad m \equiv 1 \bmod ^{*} 4 O_{E}, \quad \mathcal{C l}\left(I^{-1}\right)=C,
$$

where mod* is the usual notion of congruence in class field theory (see $[\mathrm{N}]$ ). We have

$$
(m \sigma(m) \tau(m \sigma(m))) O_{E}=(I \sigma(I) \tau(I) \tau \sigma(I))^{2} \mathfrak{P} \sigma(\mathfrak{P}) \tau(\mathfrak{P}) \tau \sigma(\mathfrak{P}) .
$$

Put $a=m \sigma(m) \tau(m \sigma(m))$. It is obvious that $a$ is not a square in $E\left(v_{\mathfrak{P}}(a) \equiv 1 \bmod 2\right)$. Let $K / k$ be the non-Galois cubic subextension of $E / k$ fixed by $\tau$. Since $\operatorname{Gal}(E / K)=\langle\tau\rangle$, we have $a=N_{E / K}(m \sigma(m))$ $\in K$. Let $M$ be the quadratic extension $K(\sqrt{a}) / K$. We have $N_{K / k}(a)=$ $\left(N_{E / k}(m)\right)^{2}$. By Lemma 2.2, $E / k$ is embeddable in the octahedral extension $N=E(\sqrt{a}, \sqrt{\sigma(a)})$.

Let $L$ be the quadratic extension $E(\sqrt{a}) / E$. We deduce from $m \equiv 1 \bmod ^{*}$ $4 O_{E}$ that $\gamma(m) \equiv 1 \bmod ^{*} 4 O_{E}$ for $\gamma=\sigma, \tau$ or $\tau \sigma$, hence $a \equiv 1 \bmod ^{*} 4 O_{E}$. By Kummer theory (see $[\mathrm{H}, \S 39]) \Delta(L / E)=\mathfrak{P} \sigma(\mathfrak{P}) \tau(\mathfrak{P}) \tau \sigma(\mathfrak{P})$. A result of Artin (see $[\mathrm{A}]$ ) yields $\mathcal{C} l_{E}\left(O_{L}\right)=\mathcal{C l}(I \sigma(I) \tau(I) \tau \sigma(I))^{-1}$, whence

$$
\mathcal{C} l_{E}\left(O_{L}\right)=C \sigma(C) \tau(C) \tau \sigma(C) .
$$

Using Proposition 2.1 we get

$$
\mathcal{C} l_{k}\left(O_{N}\right)=\left(\mathcal{C} l_{k}\left(O_{E}\right)\right)^{4}\left(N_{E / k}(C \sigma(C) \tau(C) \tau \sigma(C))\right)^{3} .
$$

Therefore

$$
\mathcal{C} l_{k}\left(O_{N}\right)=\left(\mathcal{C} l_{k}\left(O_{E}\right)\right)^{4}\left(c^{4}\right)^{3}=\left(\mathcal{C l}_{k}\left(O_{E}\right)\right)^{4} c^{3} .
$$

We conclude that (2.4) holds, and then (2.1) follows thanks to (2.3) and (2.4).

Clearly $E(\sqrt{a}) / E$ and $E(\sqrt{\sigma(a)}) / E$ are tame. It follows that $N / E$ is tame. If $E / k$ is tame, so is $N / k$. Therefore

$$
\left(\mathcal{C l} l_{k}\left(O_{E}\right)\right)^{4}\left(N_{E / k}(\mathcal{C l}(E))\right)^{3} \subset R_{t}(E / k, \Sigma) .
$$

Hence $R(E / k, \Sigma)=R_{t}(E / k, \Sigma)$, which completes the proof of $(2.2)$.

Now we complete the proof of (i). Let $k^{\prime} / k$ be the quadratic subextension of $E / k$. Because the class number of $k$ is odd, $k^{\prime} / k$ is ramified. Since it is the 
unique nontrivial abelian subextension of $E / k$, we infer that $N_{E / k}: \mathcal{C l}(E) \rightarrow$ $\mathcal{C l}(k)$ is surjective (see [W, Theorem 10.1, p. 400]). Therefore $N_{E / k}(\mathcal{C l}(E))=$ $\mathcal{C l}(k)$. Hence

$$
R(E / k, \Sigma)=\left(\mathcal{C l}_{k}\left(O_{E}\right)\right)^{4}(\mathcal{C l} l(k))^{3}=\mathcal{C} l_{k}\left(O_{E}\right)(\mathcal{C l}(k))^{3} .
$$

Proof of Theorem 1.1(ii). Let $D_{3}$ be the dihedral group of order 6. For any number field $k$, it follows from [E, Chap. III, $\S 3,3.1$, p. 59] that

$$
R_{t}\left(k, D_{3}\right)=\mathcal{C l}(k) .
$$

Let $c \in \mathcal{C l}(k)$. There exists a tame dihedral extension $E / k$ of degree 6 such that $c=\mathcal{C} l_{k}\left(O_{E}\right)$. On the other hand, by Theorem 1.1(i), $c \in R_{t}(E / k, \Sigma)$, thus $\mathcal{C l}(k) \subset R_{t}\left(k, S_{4}\right)$, whence $R_{t}\left(k, S_{4}\right)=\mathcal{C} l(k)$. Now, the equality $R\left(k, S_{4}\right)$ $=R_{t}\left(k, S_{4}\right)$ is obvious.

\section{References}

[A] E. Artin, Questions de base minimale dans la théorie des nombres algébriques, in: Colloq. Internat. CNRS 24, Paris, 1950, 19-20.

[C1] J. E. Carter, Steinitz classes of a nonabelian extension of degree $p^{3}$, Colloq. Math. 71 (1996), 297-303.

[C2] -, Module structure of integers in metacyclic extensions, ibid. 76 (1998), 191-199.

[C3] - Steinitz classes of nonabelian extensions of degree $p^{3}$, Acta Arith. 78 (1997), 297-303.

[E] L. P. Endo, Steinitz classes of tamely ramified Galois extensions of algebraic number fields, thesis, University of Illinois at Urbana-Champaign, 1975.

$[\mathrm{F}] \quad$ A. Fröhlich, The discriminant of relative extensions and the existence of integral bases, Mathematika 7 (1960), 15-22.

[FT] A. Fröhlich and M. J. Taylor, Algebraic Number Theory, Cambridge Univ. Press, 1991.

[GS] M. Godin and B. Sodaïgui, Classes de Steinitz d'extensions à groupe de Galois $A_{4}$, J. Théor. Nombres Bordeaux 14 (2002), 241-248.

[H] E. Hecke, Lectures on the Theory of Algebraic Numbers, Grad. Texts in Math. 77, Springer, New York, 1981.

[J] A. Jehanne, Sur les extensions de $\mathbb{Q}$ à groupe de Galois $S_{4}$ ou $\tilde{S}_{4}$, Acta Arith. 69 (1995), 259-276.

[Ma] J. Martinet, Discriminants and permutation groups, in: Number Theory, R. A. Molin (ed.), de Gruyter, Berlin, 1990, 359-385.

[Mc] L. R. McCulloh, Galois module structure of abelian extensions, J. Reine Angew. Math. 375/376 (1987), 259-306.

[N] J. Neukirch, Class Field Theory, Springer, Berlin, 1986.

[So1] B. Sodaïgui, Classes de Steinitz d'extensions galoisiennes relatives de degré une puissance de 2 et problème de plongement, Illinois J. Math. 43 (1999), 47-60.

[So2] - Relative Galois module structure and Steinitz classes of dihedral extensions of degree 8, J. Algebra 223 (1999), 367-378.

[So3] - Realizable classes of quaternion extensions of degree 4l, J. Number Theory 80 (2000), 304-315. 
[W] L. C. Washington, Introduction to Cyclotomic Fields, 2nd ed., Springer, Berlin, 1996.

Département de Mathématiques

Université de Valenciennes

Le Mont Houy

F-59313 Valenciennes Cedex 9, France

E-mail: marjory.godin@univ-valenciennes.fr

bouchaib.sodaigui@univ-valenciennes.fr

Received on 11.6.2001

and in revised form on 13.1.2003 